\title{
Acknowledgment to Reviewers of International Journal of Financial Studies in 2020
}

\author{
International Journal of Financial Studies Editorial Office
}

Citation: International Journal of

Financial Studies Editorial Office. 2021. Acknowledgment to Reviewers of International Journal of Financial Studies in 2020. International Journal of Financial Studies 9: 8. https:// doi.org/10.3390/ijfs9010008

Published: 24 January 2021

Publisher's Note: MDPI stays neutral with regard to jurisdictional claims in published maps and institutional affiliations.

Copyright: (C) 2021 by the author. Licensee MDPI, Basel, Switzerland. This article is an open access article distributed under the terms and conditions of the Creative Commons Attribution (CC BY) license (http://creativecommons.org/licenses /by/4.0/).

MDPI AG, St. Alban-Anlage 66, 4052 Basel, Switzerland

Peer review is the driving force of journal development, and reviewers are gatekeepers who ensure that International Journal of Financial Studies maintains its standards for the high quality of its published papers. Thanks to the cooperation of our reviewers, in 2020, the median time to first decision was 23days and the median time to publication was 57 days. The editors would like to express their sincere gratitude to the following reviewers for their precious time and dedication, regardless of whether the papers were finally published:

Agliardi, Elettra

Agnusdei, Giulio Paolo

Al-Bahrani, Abdullah

Alliata, Davide Roberto

Álvarez-Otero, Susana

Anouze, Abdel Latef

Arslan-Ayaydin, Özgür

Artene, Alin

Atukeren, Erdal

Ausloos, Marcel

Bace, Edward

Baek, Seungho

Balvers, Ronald

Barattieri, Alessandro

Barth, James R.

Baselga-Pascual, Laura

Basse, Tobias

Batrancea, Larisa

Belenioti, Zoe-Charis

Berber, Nemanja

Bernal Escoto, Blanca Estela

Biancone, Paolo

Bojnec, Stefan

Bolton, Brian

Boratyńska, Katarzyna

Bostan, Ionel

Bouri, Elie

Breeden, Joseph L

Brezeanu, Petre

Brzozowski, Jan

Burchi, Alberto

Burdekin, Richard
Busu, Mihail

Cai, Cynthia

Calin, Adrian Cantemir

Campbell, John Y.

Cepoi, Cosmin-Octavian

Cerquetti, Mara

Chang, Jung-Hsien

Chatzopoulos, Dimitris

Cheema, Muhammad A.

Chen, Hung-Ling

Cheng, Teng Yuan

Chodnicka-Jaworska, Patrycja

Choi, Moon Sub

Clark, Gordon L.

Clevenhaus, Anna

Coelho, Luís Miguel Serra

Cortis, Dominic

Cruz, Samantha

Davidavičienè, Vida

Dengov, Victor

DiLellio, James A.

Djalilov, Khurshid

Dobroszek, Justyna

Domadenik, Polona

Dospinescu, Octavian

Dreyer, Marc

Du, Wei

Durana, Pavol

Dutta, Kabir

Esparcia, Carlos

Farris, Ted

Fatoki, Olanrewaju Isola 
Fedele, Maria

Fernholz, Ricardo T.

Ferreira, Paulo

Ferretti, Paola

Filer, Randall K.

Fjesme, Sturla Lyngnes

Flores-Ruiz, David

Francis, Johanna L

Franco Martínez, Juan Agustín

Gatto, Andrea

Gebauer, Stefan

Glas, Andreas Herbert

Gogas, Periklis

González-Velasco, Carmen

Goyal, Rinkaj

Green, Jeremiah

Grigore, Ana-Maria

Guaita-Pradas, Inmaculada

Guesmi, Khaled

Harris, Becky

Hattori, Mary Therese Perez

Hauff, Jeanette

Hazell, Peter

Hendijani, Rosa

Herrera-Ballesteros, Juan

Hitzemann, Steffen

Hkiri, Besma

Huang, Cheng-Tsu

Huang, Kershen

Hung, Li-San

Huterska, Agnieszka

Huynh, Toan Luu Duc

Ivascu, Larisa

Iwański, Rafał

Janovec, Michal

Jawab, Fouad

Jindrichovska, Irena

Kapar, Burcu

Karagozoglu, AK

Kedžo, Margareta Gardijan

Keliuotytè-Staniulènienè, Greta

Kim, Seon Mi

Klimach, Anna

Koki, Constandina

Kołoszycz, Ewa

Korsakienè, Renata

Korzeb, Zbigniew

Kośny, Marek

Kosztowniak, Aneta

Kot, Sebastian

Kountzakis, Christos
Kuhn, Lena

Lamdin, Douglas J.

Larkin, Charles

Lazaroiu, George

Lee, Chih-Jen

Lee, Dongheun

Lee, Jaehyuk

Lefley, Frank

Li, Frank

Li, Kun

Lichtenstein, Benyamin

Lindblad, Fredrik

Lobao, Julio

Lucarelli, Caterina

Lyakin, Alexander

Lyhagen, Johan

Magerakis, Efstathios

Maitah, Mansoor

Mango, Fabiomassimo

Marasović, Branka

Marbaniang, Domenic

Marintseva, Kristina

Masud, Md. Abdul Kaium

Mathews, John

Matos, Pedro Verga

McMillan, David

Mehrotra, Aaron N.

Mendoza, Javier

Merker, Christopher K.

Meyer, Daniel Francois

Montanes, Antonio

Moraru, Andreea-Daniela

Motylska-Kuzma, Anna

Moutanabbir, Khouzeima

Mukerji, Purba

Mukhtarov, Shahriyar

Musa, Husam

Musile Tanzi, Paola

Nahhas, Abdulkader

Nam, Giseok

Narayan, Paresh Kumar

Nasir, Muhammad Ali

Nath, Saheli

Naumova, O. A.

Naylor, Michael

Newaz, Mohammad

Nguyen, Hung T.

Nicolini, Gianni

Nistor, Simona

Nobanee, Haitham

Nocoń, Aleksandra 
$\mathrm{Nucu}$, Anca Elena

Oh, Eun $Y$

Ohsawa, Yukio

Okabe, Yutaka

Okanović, Andrea

Olczak, Piotr

Oleksy, Paweł

Oliva, Immacolata

Orastean, Ramona

Orudzhev, Elshar

Osorio, Carlos

Ozdemir, Nilufer

Palazzo, Lucio

Pan, Shanshan

Panos, Georgios

Papadamou, Stephanos

Park, Cheong K

Park, Narang

Parnes, Dror

Parra, Jesus Mauricio Flórez

Partal Ureña, Antonio

Pereira, Cláudia Maria Ferreira

Pieńkowska-Kamieniecka, Sylwia

Podhorská, Ivana

Popescu, Madalina Ecaterina

Power, Bernadette

Prast, Henriëtte

Quoreshi, Shahiduzzaman

Qureshi, Azeem

Ramos Requena, José Pedro

Rapos, Gregory

Rathore, Heena

Reshetnikova, Liudmila G.

Rettl, Daniel

Rjoub, Husam

Röder, Klaus

Rodríguez-Moreno, Germán

Romanova, Inna

Rosch, David Michael

Rose-Ackerman, Susan

Rossi, Ludovico

Safar, Leos

Sahut, Jean-Michel

San-José, Leire

Sarno, Domenico

Scantlebury, Michael

Schlachter, Kai-Heinrich

Schmerer, Hans-Jörg

Schneider, Friedrich

Serret, Vanessa

Shen, Huawen
Sherraden, Margaret

Shih, Kuang-hsun

Sichigea, Mirela

Siedlecki, Rafal

Sirnes, Espen

Skouri, Konstantina

Škrinjarić, Tihana

Ślusarczyk, Beata

Smaga, Pawel

Smith, Christy

Sogiakas, Vasilios

Solna, Knut

Song, Suyong

Sprincean, Nicu

Sroka, Włodzimierz

Stankiewicz-Mróz, Anna

Stasiak, Michal

Stawska, Joanna

Steel, William F.

Stoica, Ovidiu

Stroup, Caleb

Sultonov, Mirzosaid

Świecka, Beata

Tan, Yong

Tang, Dr. Jian

Tang, Hongfei

Taujanskaite, Kamile

Teresienė, Deimantė

Thiagan Sinnadurai, Philip

Timochenko, Konstantin Yurievich

Tiron Tudor, Adriana

Tomić, Nenad

Trinh, Quang Vu

Trueck, Stefan

Tsoukas, Serafeim

Vagner, Ladislav

Van Der Voordt, Theo

Van Order, Robert

Vassakis, K.

Vâtcă, Anamaria

Vavrek, Roman

Vergos, Konstantinos

Wallmeier, MARTIN

Wang, Gang

Wang, Xiaoli

Warchlewska, Anna

Westerholm, Joakim

Wolniak, Radosław

Xiao, Yajun

Xu, Lei

$\mathrm{Xu}$, Randall 
$\mathrm{Xu}$, Xiaoqing Eleanor

Yao, Shu-Nung

Yohan, Alexander

Yoo, Seong-Joon

Young, Brian

Yu, Tsu-Wei
Zagaglia, Paolo

Zaremba, Adam

Zhang, Yuejun

Zimmermannová, Jarmila

Zurbruegg, Ralf 